\title{
Phylogenetic and epidemiological analysis of measles outbreaks in Denmark, 2013 to 2014
}

LD Rasmussen ${ }^{1}$, J Fonager ${ }^{1}$, LK Knudsen ${ }^{2}$, PHS Andersen ${ }^{2}$, J Rønn ${ }^{1}$, MW Poulsen ${ }^{1}$, KT Franck ${ }^{1}$, TK Fischer ${ }^{13}$

1. Microbiological Diagnostics and Virology, Statens Serum Institut, Copenhagen, Denmark

2. Department of Infectious Disease Epidemiology, Statens Serum Institut, Copenhagen, Denmark

3. Centre for Global Health, University of Southern Denmark, Odense, Denmark

Correspondence: Lasse Dam Rasmussen (LARA@ssi.dk)

Despite the introduction of safe, effective vaccines decades ago and joint global public health efforts to eliminate measles, this vaccine-preventable disease continues to pose threats to children's health worldwide. During 2013 and 2014, measles virus was introduced into Denmark through several independent importations. This resulted in a number of secondary cases $(n=7)$, with two clusters in 2013 and one in 2014. In total, there were 44 cases of measles. Most cases $(n=41)$ were laboratory confirmed by detection of measles virus genome by real-time reverse transcription (RT)-PCR and IgM antibodies. The viruses from confirmed cases were genotyped by sequencing. Only one genotype circulated each year, i.e. D8 and B3, respectively. Sequencing of measles virus from different clinical specimens from the same patients revealed that sequence variants of measles viruses might co-exist and co-transmit during an outbreak. The majority of the cases were unvaccinated $(n=27)$ or recipients of one dose of measles-mumps-rubella $(M M R)$ vaccine $(n=7)$. In addition, two fully vaccinated adult cases were reported in 2014. We demonstrate the transmission of measles virus in a population in which the two-dose MMR vaccination coverage rate was $80 \%$ and how even vaccinated individuals may be at risk of contracting measles once transmission has been established.

\section{Introduction}

Measles is caused by a negative-sense single-stranded RNA virus belonging to the Morbillivirus genus. The wild-type measles virus consists of 24 genotypes that are grouped into eight clades [1]. Measles is one of the leading causes of childhood mortality worldwide: about 145,700 people died from measles in 2013 , most of them children under the age of five years [2].

The first measles-mumps-rubella (MMR) vaccination programme was implemented in the 1960 s in the United States [3]. The World Health Organization (WHO) has set the goal of eliminating measles in Europe by 2015 and globally by 2020 [4]. In 2013, the WHO Regional Office for Europe produced a supplement for accelerated action during 2013 to 2015 [5]. In this supplement, WHO identified a number of acceleration strategies to achieve this goal by the end of 2015: (i) immunisation system strengthening; (ii) establishing and improving case-based surveillance; (iii) improving laboratory and epidemiological data integration; and (iv) improving outbreak response. These strategies have been adapted and are some of the key elements in the Danish national disease elimination efforts.

In Denmark, measles is a notifiable disease, and national measles surveillance is conducted through close collaboration between the WHO National Reference Laboratory for Measles and Rubella at the Department of Microbiological Diagnostics and Virology at the Statens Serum Institut (SSI), the Department of Infectious Disease Epidemiology, SSI, the Danish Health and Medicines Authority, general practitioners (GPs), hospital clinicians, regional hospital microbiology laboratories and public health medical officers. The public health medical officers perform contact tracing and give advice to hospitals and GPs on post-exposure prophylactic therapeutics.

In Denmark, the first dose of MMR vaccine has been given at the age of 15 months since its introduction in 1987. In 2008, the Danish Expanded Programme on Immunisation (EPI) schedule was changed and the timing of the second MMR vaccine dose adjusted from 12 years to 4 years of age. The rationale for the change was to increase herd immunity by increasing the immunity of children aged under 12 years [6].

Since 1996, MMR vaccination status in Denmark has been recorded in a national vaccination database, in which data are automatically entered when GPs use specific codes for reimbursement for performing the 
New measles notifications by week in Denmark, 2013 and $2014(\mathrm{n}=44)$

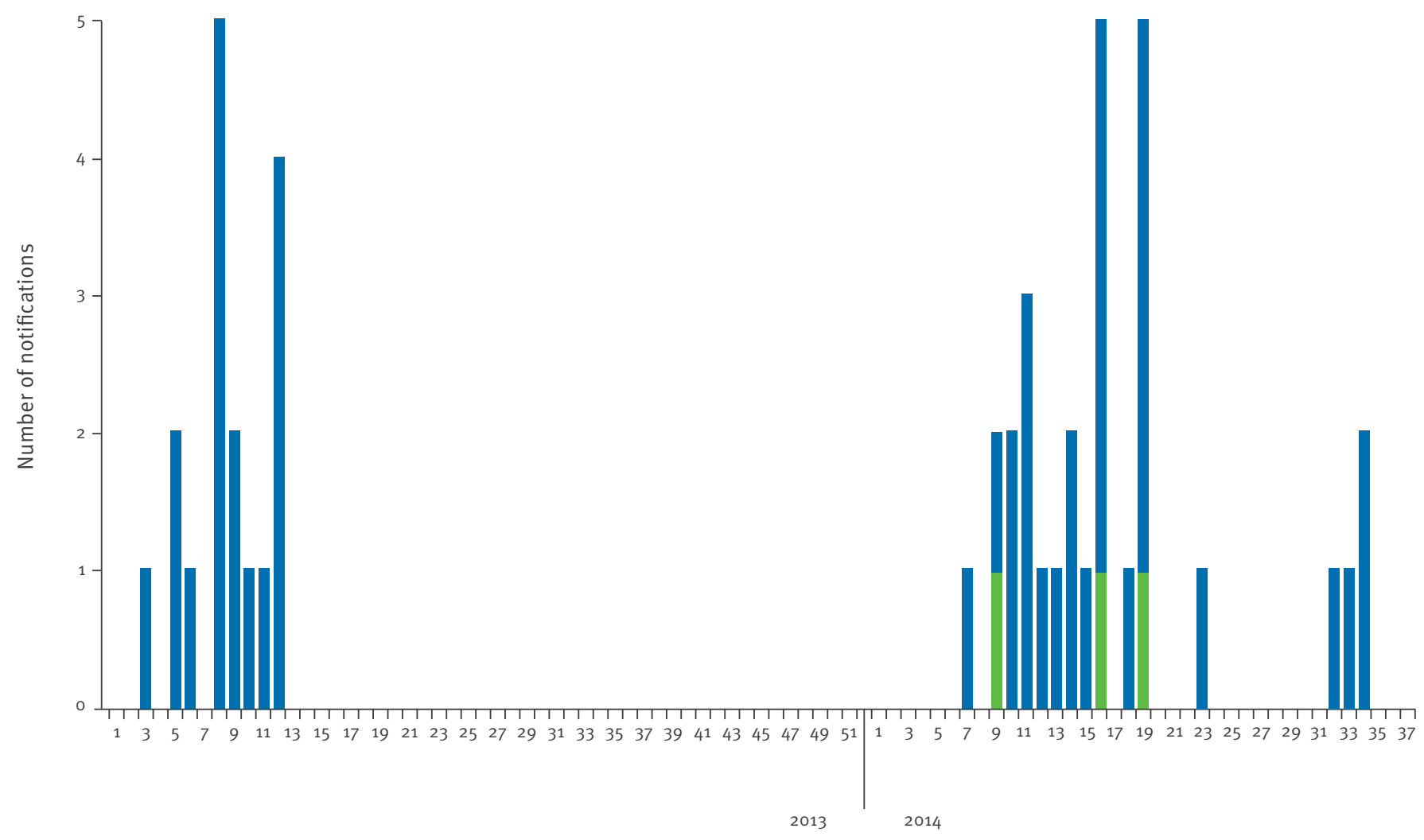

Week number

For the 41 laboratory-confirmed cases (shown in blue), the notification date is based on first sampling day. For the three probable cases, whose infection was not laboratory confirmed but diagnosed based on clinical criteria and epidemiological link (shown in green), GP diagnosis date is used.

For 2014, only weeks 1-37 are shown, but no cases were notified during the rest of the year.

vaccinations. Since the introduction of this database, MMR vaccination coverage in Denmark has been estimated to be $88-90 \%$ for the first dose and $83-88 \%$ for the second [7]. That is until 2013, when coverage decreased to $87 \%$ for the first dose and $80 \%$ for the second, underlining the challenge facing the Danish EPI programme when trying to meet the goal of $95 \%$ coverage, as recommended by WHO [8]. However, a study in which parents of children not registered in the national database were interviewed about the vaccination status of their children documented under-reporting of coverage in the database, to the magnitude of 3-4 percentage points [7]. Given this, and the fact that the vaccination of some of children will be delayed [7], coverage may be higher in reality.

The advantage of molecular characterisation of measles virus followed by phylogenetic analysis during outbreaks has been demonstrated previously $[9,10]$. In our current study, we combine phylogenetic analyses of viruses from confirmed measles cases in Denmark during 2013 and 2014 with classical epidemiological investigations in order to investigate the consequences of imported cases of measles and the associated potential public health challenges in the attempt to keep a nation free of endemic transmission of measles virus.

\section{Methods}

We used the European Union measles case definition, as described by ECDC [11]. We defined a cluster as having a minimum of three persons with measles, for whom an epidemiological link to an identified index case, either directly or by secondary infection, was established.

For any measles case, the local GP takes the lead on collecting all available information on the patient that is necessary for public health action, such as the likely source of exposure and contacts of the case. This information is recorded in a standardised notification Form (National Board of Health form 1515), which is sent to both the Department of Infectious Disease Epidemiology at SSI and to the Regional State Epidemiologist (RSE). After receiving the official notification form, the RSE decides on the appropriate public health action, e.g. tracing unvaccinated contacts and the extent of administration of prophylactic vaccination or immunoglobulin. 
Possible transmission routes in clusters of measles cases in Denmark, 2013 and $2014(\mathrm{n}=17)$

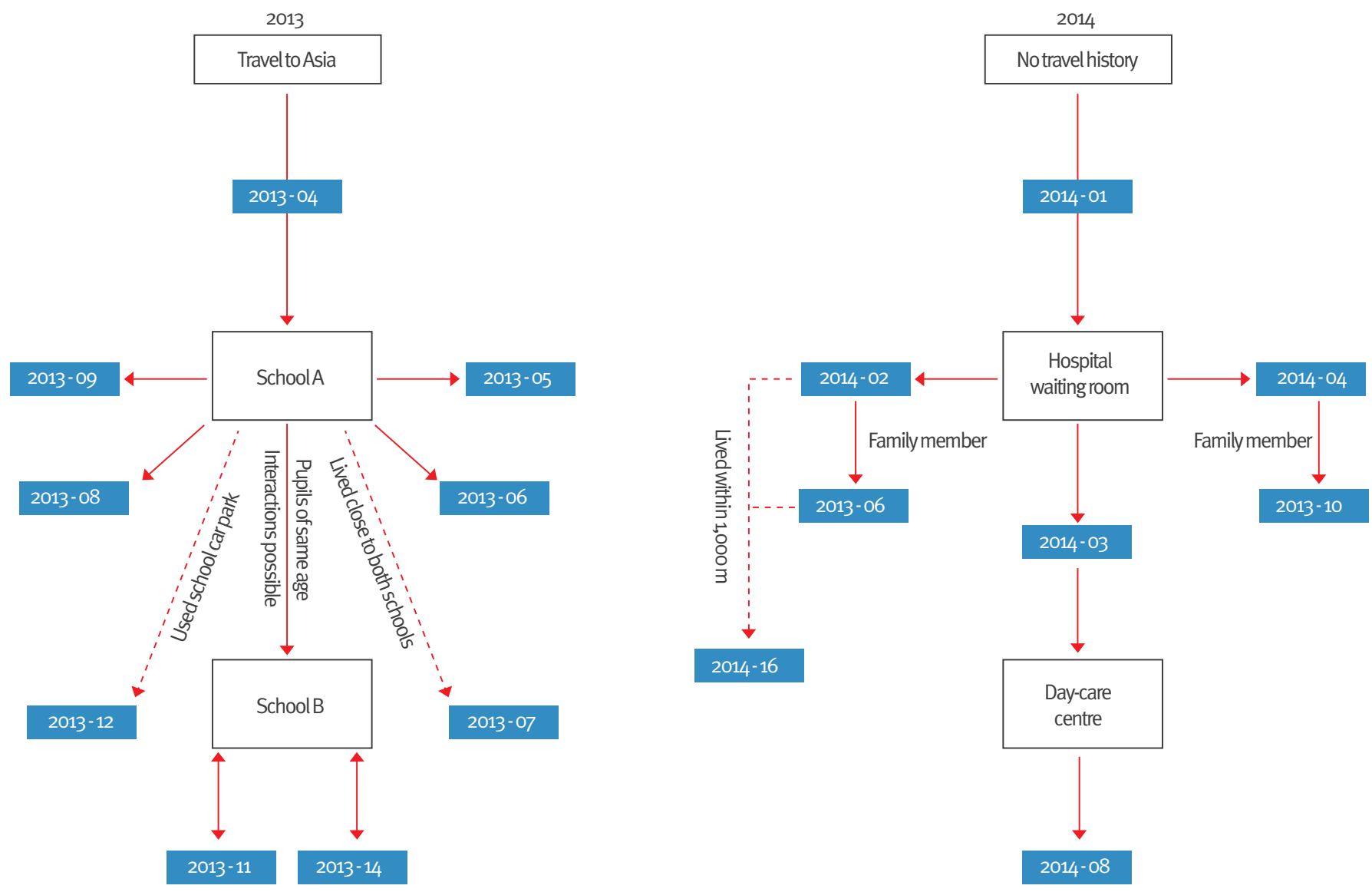

For 2013 , only the large cluster is shown. Solid lines show epidemiological links involving known close contact with a measles case, whereas dotted lines indicate possible contact. The arrows show the direction of transmission. Double-headed arrows indicate a possible connection between the cases but the direction of the transmission cannot be established.

\begin{abstract}
Analysis of specimens
During 2013 to 2014 , specimens from suspected measles cases - primarily urine, throat swabs and serum were sent to the WHO National Reference Laboratory for Measles and Rubella, SSI, for laboratory confirmation and further characterisation of measles virus-positive samples. In most cases, multiple specimens were submitted simultaneously. Serum specimens were tested for IgG and IgM antibodies using a BEP 2000 Advance system (Siemens Healthcare) with an in-house ELISA (unpublished data). Measles virus RNA was purified using the MagNA pure LC system (Roche), using the Total NA kit (Roche).
\end{abstract}

All samples (serum, throat swabs and urine) were further tested for the presence of measles virus RNA by real-time reverse transcription-PCR (RT-PCR) according to the procedures described by Mubarak et al. [12]. Positive samples were further characterised by RT-PCR followed by Sanger sequencing on an ABI 3100 (Thermo Fischer) using primers and running conditions as recommended by WHO [13]. Measles virus sequences were genotyped online using the $450 \mathrm{bp}$ fragment of the $\mathrm{N}$ gene recommended for genotyping by WHO by the shared member state sequence database, the Measles Nucleotide Surveillance (MeaNS) tool [14].

Phylogenetic analyses were performed by alignment and neighbour-joining tree-building using ClustalW [15]. Bootstrapping was performed using 1,000 replicates. Sequences shorter than the recommended 450 bp were excluded from the phylogenetic analysis.

\section{Vaccination status}

Vaccination status was based on data available in the national vaccination database, which was initiated in 1996; status before this was based on statements from the patient and/or the GP. Thus, vaccination status was only fully available in the database for persons up to 17 years and 18 years of age, in 2013 and 2014, respectively.

\section{Nomenclature}

Confirmed cases were recorded using the year and chronological order in which they were laboratory confirmed (e.g. the first case in 2013 was given the ID 201301). All viral sequences were named in accordance with the WHO standards implemented by MeaNS [14]. 
FIGURE 3

Measles cases by age group, Demark, 2013 and 2014 ( $\mathrm{n}=$ 44)

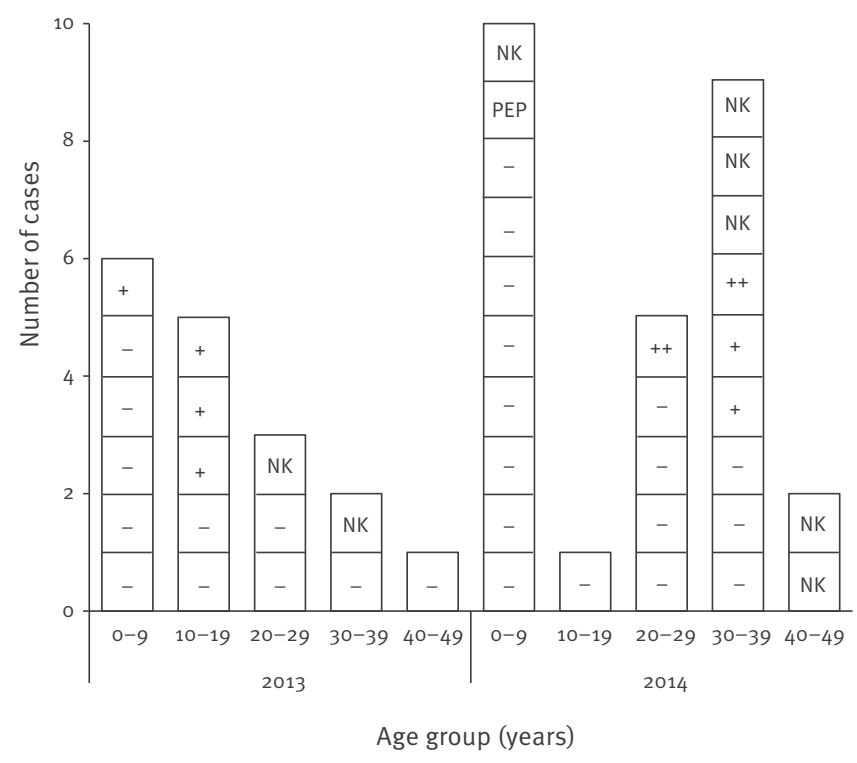

MMR: measles-mumps-rubella; NK: unknown; PEP: post-exposure prophylaxis; RT-PCR: real-time reverse transcription-PCR.

Vaccination status: - indicates that the person was not vaccinated with a measles virus-containing vaccine; + indicates that the person had received the first dose of MMR vaccine; ++ indicates that the person had received both doses of MMR vaccine.

\section{Results}

Epidemiology of measles cases in Denmark in 2013 and 2014

In 2013 and 2014, 17 and 27 measles cases were notified, respectively. In 2013, all cases were laboratory confirmed, whereas in 2014, 24 cases were laboratory confirmed, while GPs (one in France and two in Denmark) diagnosed the remaining three probable cases, based on clinical and epidemiological criteria.

In 2013, the cases were notified during weeks 3 to 12 , whereas in 2014, the first notification came slightly later, but the cases were notified over a longer period (weeks 7-34) (Figure 1). In the latter cases, diagnosis was based on clinical signs and epidemiological links to known laboratory confirmed cases. For a detailed overview of all cases from both years, see the Table.

In 2013, there was a large cluster consisting of nine epidemiologically linked cases (case ID 2013-04 to 201309, 2013-11, 2013-12 and 2013-14) and a small cluster of four cases (2013-13 and 2013-15 to 2013-17). An additional four unlinked cases were detected: three of these cases (2013-01 to 2013-03) were notified before the first cluster was identified. One had travelled from the United Kingdom and one from Asia during the incubation period; the source of the infection of the third was unknown. The only other unlinked case (2013-10), occurred during the same time as the cases in the large cluster and was of the same age group as the majority in the cluster (7-11 years), but no epidemiological link could be established.

During 2014, a single cluster was detected, consisting of eight cases (2014-01 to 2014-04, 2014-06, 2014-08, 2014-10 and 2014-16). In addition, five imported cases each resulted in one secondary case (2014-05 linked to 2014-07, 2014-09 linked to 2014-11, 2014-12 linked to 2014-14, 2014-15 linked to 2014-20, and 2014-19 linked to 2014-22) and one importation (2014-24) resulted in two secondary cases (2014-25 and 2014-27). The remaining six cases were regarded as individual importations for whom no epidemiological links could be established. The cases for whom the travel history was known showed travel to Asia (2014-05 and 2014-12), the Netherlands and Germany (2014-13) and Turkey (2014-24). One case, an asylum seeker (2014-26), had been travelling through Europe, but the sequence of the measles virus from that case grouped together with that from the case who had travelled to Turkey (see below).

In 2013, the first measles case was identified in midJanuary as an imported case from the United Kingdom. Within two weeks, two other unconnected cases were confirmed, one imported from Asia and one with no travel history. The index case of the 2013 large cluster was diagnosed with measles during the first week of March. The patient (2013-04), aged 10-19 years, had returned to School A after a trip to Asia. In the same school, four other pupils were also diagnosed with measles within the incubation period for the disease.

Two weeks after the introduction of measles at School A, a child at another school (School B), comprising pupils of the same age group, located less than 200 metres from School A, was diagnosed with measles and two weeks later, another child was diagnosed with measles at School B. In addition to the seven pupils from the two schools, this cluster also contained two individuals with a possible epidemiological link to the cluster: a person in their 30 s who used the car park at School A and a person in their 40 s who lived close to both schools (Figure 2).

The second smaller measles cluster occurred in a daycare centre, in the same town as the larger cluster, involving three children aged under 4 years and a person in their $20 \mathrm{~s}$ (Table). Even though direct epidemiological links between this and the large cluster could not be established, sequence homology of the measles viruses and the close proximity in time and location suggest a link between the two clusters. The remaining case from 2013 (2013-10), even though no epidemiological link could be established, were connected to the same town; thus 14 of the 17 cases in 2013 were relatively closely linked geographically.

The index case of the 2014 cluster (2014-01) was an unvaccinated child aged under four years with no known travel history outside Denmark. Before being 


\section{FIGURE 4}

Neighbour-joining tree showing the phylogenetic relationship of measles virus sequences from cases in Denmark, 2013 and 2014

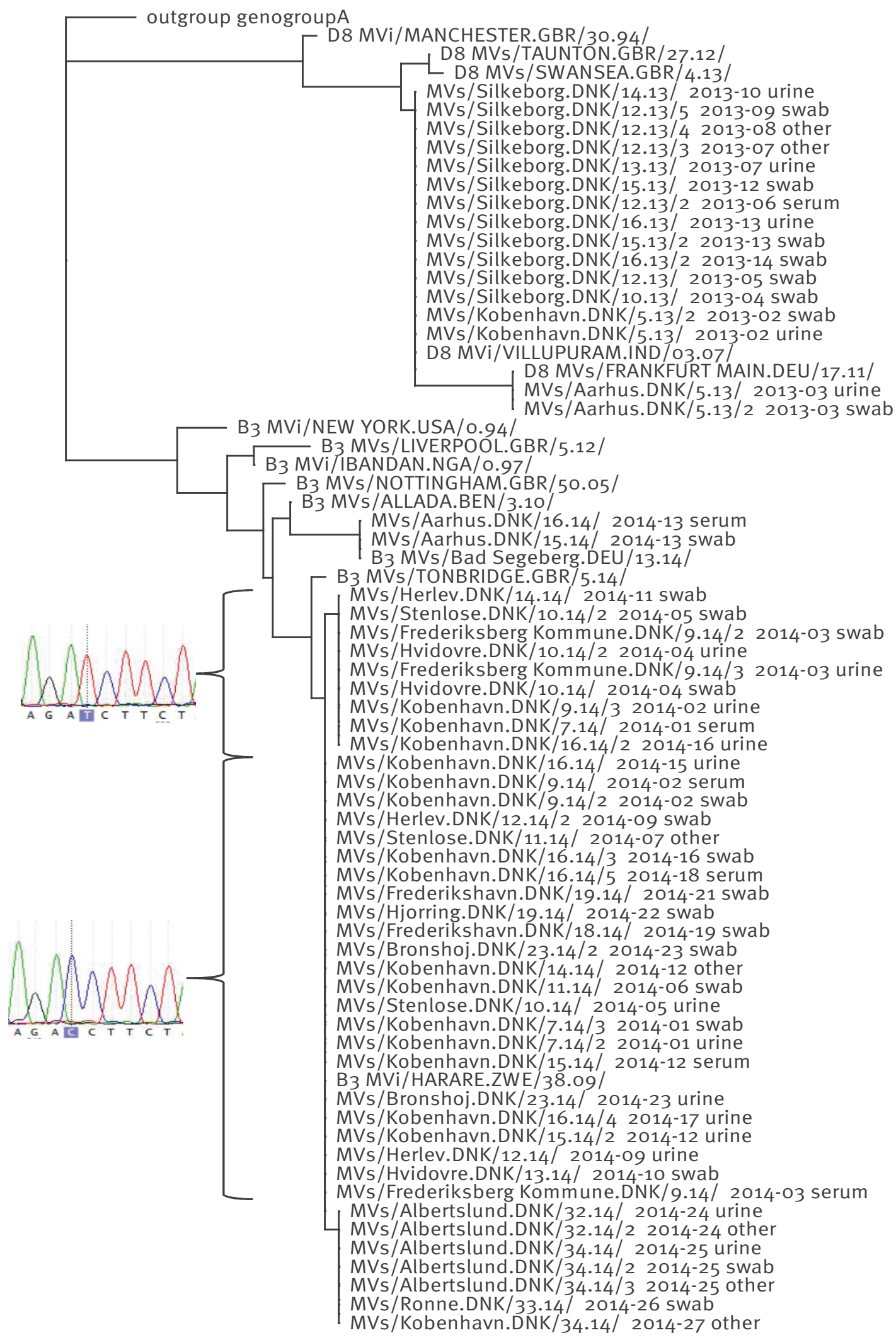

Only sequences consisting of the recommended $450 \mathrm{bp}$ of the measles virus $\mathrm{N}$ region were included in the analysis ( 57 sequences from 36 cases). Bootstrapping was performed using 1,000 replicates (for clarity, bootstrap values are omitted). The names of the sequences consist of the name assigned by MeaNS followed by the case ID and the type of specimen. Reference strains show the genotype followed by the name of the reference strain in capital letters.

The chromatograms showing the conserved single base mutation between the two Bz clades is included to show the unambiguity of the sequencing results. 
TABLE

Overview of all cases of measles in Denmark, 2013 and $2014(\mathrm{n}=44)$

\begin{tabular}{|c|c|c|c|c|c|c|c|c|}
\hline \multirow[b]{2}{*}{ Case ID } & \multirow{2}{*}{$\begin{array}{c}\text { Age } \\
\text { group } \\
\text { in years }\end{array}$} & \multicolumn{4}{|c|}{ RT-PCR-positive sample } & \multirow{2}{*}{$\begin{array}{l}\text { Vaccination } \\
\text { status }\end{array}$} & \multirow{2}{*}{$\begin{array}{c}\text { First } \\
\text { sampling } \\
\text { date }^{\mathrm{b}}\end{array}$} & \multirow[b]{2}{*}{ Epidemiological link } \\
\hline & & Serum & Urine & $\begin{array}{l}\text { Throat } \\
\text { swab }\end{array}$ & Unknown ${ }^{a}$ & & & \\
\hline 2013-01 & $20-29$ & & & & & - & $17 \operatorname{Jan} 2013$ & Travel to United Kingdom \\
\hline 2013-02 & $30-39$ & & & & & + & $31 \operatorname{Jan} 2013$ & Travel to Asia \\
\hline 2013-03 & $20-29$ & & & & & - & 1 Feb 2013 & Unknown \\
\hline 2013-04 & $10-19$ & & & & & + & 8 Mar 2013 & Index case of large cluster (stay in Asia) \\
\hline $2013-05$ & $10-19$ & & & & & + & 18 Mar 2013 & 2013-04 (attended School A) \\
\hline 2013-06 & $10-19$ & & & & & - & 19 Mar 2013 & 2013-04 (attended School A) \\
\hline 2013-07 & $40-49$ & & & & & - & 21 Mar 2013 & Lived close to Schools A and B \\
\hline $2013-08$ & $5-9$ & & & & & - & 21 Mar 2013 & 2013-04 (attended School A) \\
\hline 2013-09 & $5-9$ & & & & & + & 22 Mar 2013 & 2013-04 (attended School A) \\
\hline $2013-10$ & $5-9$ & & & & & - & 2 Apr 2013 & Unknown \\
\hline $2013-11$ & $10-19$ & & & & & + & 2 Apr 2013 & Classmate of 2013-14 at School B \\
\hline 2013-12 & $30-39$ & & & & & Unknown & 8 Apr 2013 & Used car park at School A \\
\hline $2013-13$ & $0-4$ & & & & & - & 14 Apr 2013 & Index case of small cluster (source of infection unknown) \\
\hline 2013-14 & $10-19$ & & & & & - & 18 Apr 2013 & Classmate of 2013-11 at School B \\
\hline 2013-15 & $0-4$ & & & & & - & 19 Apr 2013 & $\begin{array}{c}2013-13 \\
\text { (attended Day-care centre A) }\end{array}$ \\
\hline 2013-16 & $0-4$ & & & & & - & 20 Apr 2013 & $\begin{array}{l}\text { 2013-13 } \\
\text { (attended Day-care centre A) }\end{array}$ \\
\hline 2013-17 & $20-29$ & & & & & Unknown & 22 Apr 2013 & Worked at Day-care centre $\mathrm{A}$ \\
\hline 2014-01 & $0-4$ & & & & & - & 12 Feb 2014 & Index case of single cluster (source of infection unknown) \\
\hline 2014-02 & $0-4$ & & & & & - & 25 Feb 2014 & 2014-01 (in hospital waiting room when 2014-01 present) \\
\hline 2014-03 & $0-4$ & & & & & - & 28 Feb 2014 & 2014-01 (in hospital waiting room when 2014-01 present) \\
\hline 2014-04 & $0-4$ & & & & & - & 4 Mar 2014 & 2014-01 (in hospital waiting room when 2014-01 present) \\
\hline 2014-05 & $30-39$ & & & & & + & 5 Mar 2014 & Travel to Asia with 2014-07 \\
\hline 2014-06 & $0-4$ & & & & & PEP & 10 Mar 2014 & 2014-02 (family member) \\
\hline 2014-07 & $30-39$ & & & & & + & 10 Mar 2014 & Travel to Asia with 2014-05 \\
\hline 2014-08 & $0-4$ & \multicolumn{4}{|c|}{ Not sampled } & - & 14 Mar 2014 & $\begin{array}{l}\text { 2104-03, attended same day-care centre as 2104-03. } \\
\text { Diagnosed in France. }\end{array}$ \\
\hline 2014-09 & $20-29$ & & & & & - & 20 Mar 2014 & Unknown source of infection \\
\hline 2014-10 & $20-29$ & & & & & ++ & 26 Mar 2014 & 2014-04 (family member) \\
\hline 2014-11 & $30-39$ & & & & & ++ & 1 Apr 2014 & 2014-09 (family member) \\
\hline $2014-12$ & $40-49$ & & & & & Unknown & 5 Apr 2014 & Travel to Asia \\
\hline 2014-13 & $20-29$ & & & & & - & 12 Apr 2014 & Travel to Netherlands and Germany \\
\hline 2014-14 & $0-4$ & \multicolumn{4}{|c|}{ Not sampled } & - & 16 Apr 2014 & 2014-12 (relative) \\
\hline 2014-15 & $30-39$ & & & & & - & 20 Apr 2014 & Unknown source of infection \\
\hline 2014-16 & $30-39$ & & & & & - & $18 \mathrm{Apr} 2014$ & Lived near 2014-02 and 2014-06 \\
\hline 2014-17 & $20-29$ & & & & & - & 16 Apr 2014 & Unknown source of infection \\
\hline 2014-18 & $10-19$ & & & & & - & 17 Apr 2014 & Unknown source of infection \\
\hline 2014-19 & $20-29$ & & & & & - & 28 Apr 2014 & Unknown source of infection \\
\hline 2014-20 & $1-5$ & \multicolumn{4}{|c|}{ Not sampled } & - & 5 May $2014^{\mathrm{b}}$ & 2014-15 (family member) \\
\hline 2014-21 & $30-39$ & & & & & Unknown & 9 May 2014 & Unknown source of infection \\
\hline $2014-22$ & $30-39$ & & & & & - & 9 May 2014 & Worked at hospital where 2014-19 was hospitalised \\
\hline $2014-23$ & $0-4$ & & & & & - & 3 Jun 2014 & Unknown source of infection \\
\hline 2014-24 & $30-39$ & & & & & Unknown & 4 Aug 2014 & Travel to Turkey \\
\hline 2014-25 & $30-39$ & & & & & Unknown & 19 Aug 2014 & 2014-24 (family member) \\
\hline $2014-26$ & $0-4$ & & & & & Unknown & 16 Aug 2014 & Sought asylum in Denmark \\
\hline $2014-27$ & $40-49$ & & & & & Unknown & 20 Aug 2014 & $2014-24$ (visited 2014-24 in hospital) \\
\hline
\end{tabular}

MMR: measles-mumps-rubella; PEP: post-exposure prophylaxis; RT-PCR: real-time reverse transcription-PCR.

Green cells in the column 'RT-PCR-positive sample، indicate a positive diagnostic real-time RT-PCR from the specimen specified. The grey cells in this column indicate that the particular sample type was not submitted.

Vaccination status: - indicates that the person was not vaccinated with a measles virus-containing vaccine; + indicates that the person had received the first dose of MMR vaccine; ++ indicates that the person had received both doses of MMR vaccine.

The colour coding in the column 'Epidemiological link' indicates the possible links.

a Type of specimen unknown (not specified or information unobtainable for other reasons).

${ }^{b}$ Or date of diagnosis for probable cases, based on clinical signs and epidemiological link. 
admitted to the paediatric ward of a hospital, the child had stayed in two different waiting rooms: first at the GP's practice and subsequently at the hospital. The public health medical officer later determined via contact tracing that about 60 children had been in the same waiting rooms at some point during the presence of the infected child. Information about post-exposure prophylaxis to non-vaccinated contacts was sent out by post by the public health medical officer, but for some contacts, the information was received too late to prevent infection (e.g.more than 3-6 days post exposure). Subsequently, three (2014-02 to 2014-04) of the 60 children (attack rate: $5 \%$ ), were diagnosed with measles, which was confirmed both serologically and by RT-PCR. Two of the cases infected in the hospital waiting room transmitted the virus to family members, one of whom was in their 20s. Moreover, before being diagnosed with measles, one of the children who had been in the waiting room attended a day-care centre, where at least one other child was infected. All but one of these cases were aged under 4 years. Additionally, a person in their 305 (2014-16) livingless than 1,000 metres from cases 2014-02 and 2014-06 was considered part of this cluster due to geographical proximity, giving a total of eight cases in this cluster.

Three of the four later cases in 2014 (2014-24, 2014-25 and 2014-27) were likely connected, in which 2014-25, a member of 2014-24's family, as well as an acquaintance (2014-27) were infected, likely while visiting 201424 during their hospital admission for measles.

\section{Age distribution and vaccination status}

The age groups affected differed markedly between the two years. In 2013, the majority of the measles cases $(11 / 17)$ were younger than 12 years (Figure 3), whereas the majority of cases (16/27) in 2014 were older than 19 years.

Most of the 2013 and 2014 cases were unvaccinated (10/17 and 17/27, respectively). In Denmark, the twodose MMR vaccination programme was implemented in 1987. Thus, vaccination can be expected only in individuals from 15 months to 27 years of age. In this study, 13 of the 27 unvaccinated cases were outside this age group.

In seven cases, only administration of the first dose of MMR vaccine could be documented ( $n=5$ and $n=2$ in 2013 and 2014, respectively). In 2014, two persons (2014-10 and 2014-11, in their 205 and 30s, respectively) were infected in spite of having followed the full vaccination programme (Figure 3). In both cases, measles was diagnosed based on clinical symptoms and virus detection by RT-PCR from throat swabs. In neither of these cases could antibody levels or IgG avidity be tested since serum was not submitted.

For the remaining cases in both years, no vaccination history could be identified in the records of the national vaccination database and/or of the $G P(n=2$ and $n=6$, respectively) (Figure 3).

In 2014, a child aged under four years (2014-06, relative of 2014-02) received post-exposure vaccination, but became symptomatic two weeks after the initial symptoms of 2014-02.

\section{Genotyping and phylogenetic analysis}

In 2013, the only measles virus genotype identified was D8. Phylogenetic analysis showed that all sequences analysed (16 from 12 cases), except for two from the same patient (2013-03), were $100 \%$ identical to the D8 reference strain $\mathrm{MVi} /$ Villupuram.IND/03.07/. The two virus sequences from 2013-03 (from urine and swab samples) matched $100 \%$ to the D8 reference strain MVs/Frankfurt Main.DEU/17.11 (Figure 4).

In 2014, again only a single genotype was found: B3 (41 sequences from 24 cases). The majority of sequences (23/41) grouped with the B3 reference strain MVi/ Harare.ZWE/38.09/, showing $100 \%$ sequence identity. Another cluster contained nine $100 \%$ identical sequences from seven cases (Figure 4). The sequences in this cluster differed from the Harare strain by only a single conserved point mutation (Figure 4). In five cases (2014-01, 2014-02, 2014-03, 2014-05 and 201416), the sequence variants were observed in different specimens collected from the same patient, indicating that virus representing both sequences was transferred simultaneously. In all cases, only one of the two variants was detected in individual specimens (Figure 4). Analysis revealed that this is a silent mutation, not altering the amino acid sequence.

One sequence (from case 2014-13) clustered at some distance from all known B3 strains. This case was a person in their 305 who had travelled from the Netherlands via Germany to Denmark and became symptomatic after arriving home. The viral sequences from this case matched $100 \%$ with a sequence found in the MeaNS database from a measles case in Germany in 2014 (Bad Segeberg/DEU/13.14, GenBank accession number: KJ769091).

Another interesting observation is that the last four cases in 2014 (weeks 32-34, 4-20 August) grouped together. Case $2014-24$ is thought to have imported measles from Turkey and probably infected two secondary cases (2014-25 and 2014-27). The last case in this sequence group was a child seeking asylum in Denmark after travelling through Europe. The sequences in this group also differ from the B3 MVi/Harare.ZWE/38.09/ reference strain by a single silent base mutation.

\section{Discussion}

In 2012, there were two measles cases reported in Denmark [16], whereas in 2013 and 2014, 17 and 27 measles cases were notified, respectively. In these two years, only a single genotype was detected: D8 and $B_{3}$, respectively, which corresponds well with the 
genotypes dominating in Europe during these years $[17,18]$.

The pattern of spread of measles in Denmark was very similar in the two years described here. In both years, a single case resulted in a large cluster. In 2013, an additional small cluster of four cases was observed in a day-care centre, and even though no epidemiological link could be established to the large cluster, the geographical and timely coincidence between the two clusters indicates that they could be a result of the same importation. If this is true, all cases in 2013 arose from just four importations, of which three were without secondary cases. Our study demonstrates that the importation of measles virus only sometimes leads to a cluster, most likely if the virus is imported by children of pre-vaccination age.

The period in which new measles cases were detected was more prolonged in 2014 and included 10 more cases than in 2013. This may be a result of the slightly different importation pattern observed during 2014, where at least eight independent importations were detected. Of these, one resulted in the large cluster of eight cases and five cases resulted in one or two secondary cases (Table).

The 2014 cluster shares many similarities with earlier reported outbreaks in Denmark during 2008 and 2009, which were believed to have started in GPs' waiting rooms $[19,20]$. This emphasises the need to rethink how to assemble potentially contagious people waiting to be examined and their relatives in small rooms - not only in hospital settings but throughout the entire healthcare system. In Denmark, there is pre-screening before patients are referred to the hospital emergency room, either by the GP or by telephone interview by specially trained nurses outside of GP opening hours. If patients with symptoms of contagious diseases are referred to an isolation waiting room at this stage, situations like that in 2014 may be prevented. Another lesson to be learned from the 2014 outbreak is that when trying to reach traced contacts who may have been exposed to the pathogen (e.g. measles virus in this instance), using postal mail services may not be the most appropriate approach. In this digitalised era, other methods, such as a bulk text message, could have been considered, since most people own and carry a mobile phone and text messages are independent of people being able to answer the phone at a specific time.

The phylogenetic analysis in our study showed that despite the fact that multiple importations occurred from different countries, the measles viruses detected were very closely related. This may be a result of very low mutation rates found in the genome of measles virus [21]. An interesting finding was that in 2014, measles virus sequences obtained from different specimens collected from the same patient showed conserved sequence differences in several cases. Of the seven cases infected with virus containing the mutation, five
(2014-01 to 2014-04 and 2014-16) were epidemiologically connected to the large cluster (Figure 2), which supports the theory of simultaneous transfer of the two variants. This strongly indicates that different measles variants can co-exist and co-transfer simultaneously. How widespread this phenomenon is, is unknown since the MeaNS database only allows the uploading of one sequence per sample and does not allow the submission of ambiguous sequences. Furthermore, the result of Sanger sequencing is a consensus sequence: thus minority variants below the level of detection by Sanger sequencing (commonly estimated to be around $20 \%$ ) are not reported. This finding could have important implications for the use of molecular data in cluster definitions. Cases might be discarded as not being epidemiologically linked if sequencing was only performed on one specimen. Deep sequencing using next-generation sequencing technology could be used to further elucidate the prevalence of co-existing sequence variants, and to epidemiologically link the transmission of such variants.

Our data from 2013 and 2014 clearly show that the vaccination coverage of the MMR vaccine in Denmark (in 2013, it was $87 \%$ and $80 \%$ for the first and the second vaccination dose, respectively) is not high enough to prevent importation of measles leading to an outbreak due to insufficient herd protection or immunity. The data also indicate that the age of the index patient is important, if a single case is to result in an outbreak.

In both years, the cluster index cases were children who had contact with many other children of the same age. Especially in 2014, very young children, 12 months of age or younger, led to the establishment of the outbreaks. It has been argued that earlier administration of the first dose of MMR vaccine would lessen the risk of this type of outbreak [22]. A previous study has shown that vaccine failure after the second dose is significantly higher if the first dose is given to children of naturally infected mothers at $\leq 15$ months of age [23]. However, another study has shown that this may not be relevant in a population with a high vaccination coverage, since maternal antibodies from vaccinated mothers wane much faster than those from naturally infected mothers. Thus, infants are only covered for around three months by maternal antibodies from vaccinated mothers [24]. Our results show that the second vaccination dose is important for sustained protection against infection, since at least seven cases were patients of an age at which the second MMR dose should have been administered. During the two years, seven persons contracted measles after having received only the first MMR vaccine dose. This corresponds with other findings that receiving the second dose is more important than the interval between the two doses $[25,26]$. Even if the two-dose vaccination programme is adhered to, vaccine failure can be observed. In the present study, with the reservations mentioned in the results section, two cases (in their 205 and 30s) had received both MMR doses before infection. The probability of finding 
cases among vaccinated individuals will increase with increasing vaccination coverage and others have also shown vaccine failure and even further transmission from persons with documented two-dose vaccinations [27]. Both cases in our study who had received both vaccination doses (2014-10 and 2014-11) were secondary cases and did not result in further transmission. Studies have shown that in areas with no endemic measles virus circulation, antibody titres will decline over time [28]. This might be one explanation for the occurrence of measles in two-dose vaccinated cases in our study. On the other hand, caution is needed when vaccination status is based on the memory of the individuals concerned.

The effect of a third dose of MMR vaccine given at the age of 11 to 17 years has so far only been evaluated for mumps, but with promising results [29]. Whether this is also true for measles needs to be investigated. Regardless, the two-dose vaccination programme has, from its onset in Denmark in 1987, had an enormous effect on the incidence of measles. Until 1987, between 20,000 and 70,000 cases per year were not uncommon, and the mean number of measles-related deaths were one per 300 cases from 1877 to 1986 [16]. The last recorded death in Denmark caused by measles was in 1989 [16].

Of the 44 cases during 2013 to 2014 , only seven were of a pre-vaccination age, whereas 27 were unvaccinated. Seven cases received only the first dose but not the second. This shows that follow-up regarding missed vaccination, e.g. in the form of reminder letters, may be necessary in order to ensure sufficient vaccination coverage. If the WHO goal of elimination of the disease by the end of 2015 is to be achieved, new measures are needed to ensure sufficient vaccination coverage. These could include closer contact between parents and health authorities and clearer communication about vaccination, including risk-benefit and responsibility aspects. In Denmark, interviews of parents showed that the most common reason for failure to vaccinate is forgetfulness [7]. Initial experience in Denmark with reminder letters to parents of children not registered with one or more vaccinations and updated information to parents reluctant to let their children be vaccinated against MMR shows promising results [30].

\section{Conflict of interest}

None declared.

\section{Authors' contributions}

LKK, PHSA, KTF and TKF contributed to acquisition of data. JR and MWP performed laboratory work. LDR, JF, LKK, PHSA, JR, MWP and TKF analysed and interpreted the data. LDR drafted the manuscript. LDR, JF, LKK, PHSA, JR, MWP, KTF and TKF critically revised the manuscript. All authors approved the final version of the manuscript.
References

1. Measles virus nomenclature update: 2012. Wkly Epidemiol Rec. 2012;87(9):73-81.PMID: 22462199

2. World Health Organization (WHO). Measles Fact sheet no 286. Feb 2015. Geneva: WHO. [Accessed 2 Feb 2015]. Available from: http://www.who.int/mediacentre/factsheets/fs286/en/

3. SencerDJ, DullHB, LangmuirAD. Epidemiologic basis for eradication of measles in 1967.Public Health Rep. 1967;82(3):253-6. DOI: 10.2307/4592985 PMID: 4960501

4. World Health Organization (WHO). Global measles and rubella strategic plan 2012-2020. Geneva: WHO; 2012. Available from: http://apps.who.int/iris/ bitstream/10665/44855/1/9789241503396_eng.pdf

5. World Health Organisation (WHO) Regional Office for Europe. Measles and rubella elimination 2015. Package for accelerated action: 2013-2015. Copenhagen: WHO Regional Office for Europe; 2013. Available from: http://www.euro. who.int/_data/assets/pdf_file/0020/215480/PACKAGE-FORACCELERATED-ACTION-20132015.pdf?ua $=1$

6. Bottiger B, Nielsen LP. MFR 2-vaccination fremrykkes til 4 års alderen. [MMR 2 vaccination moved forward to 4 years of age]. EPI-NYT. Cophenhagen: Statens Serum Institut; 2008. Available from: http://www.ssi.dk/ /media/Indhold/DK\%20 -\%20dansk/Aktuelt/Nyhedsbreve/EPI-NYT/2008/2008\%20 PDF/EPI-NYT\%20-\%202008\%20-\%20uge\%209.ashx

7. WójcikOP, SimonsenJ, MølbakK, Valentiner-BranthP. Validation of the 5-year tetanus, diphtheria, pertussis and polio booster vaccination in the Danish childhood vaccination database. Vaccine. 2013;31(6):955-9. DOI: 10.1016/j.vaccine.2012.11.100 PMID: 23246264

8. World Health Organization Regional Office for Europe (WHO Europe). Surveillance guidelines for measles, rubella and congenital rubella syndrome in the WHO European Region. WHO publication EUR/08/5082738. Copenhagen: WHO Europe; 2009. Available from: http://www.euro.who.int/_data/assets/ pdf_file/0018/79020/E93035.pdf

9. MuscatM, VinnerL, ChristiansenAH, GlismannS, BöttigerBE. The benefit of molecular characterization during a measles upsurge in Denmark.Vaccine. 2007;25(33):6232-6. DOI: 10.1016/j.vaccine.2007.05.063 PMID: 17629377

10. VainioK, SteenTW, ArnesenTM, RønningK, ÅnestadG, DudmanS. Measles virus genotyping an important tool in measles outbreak investigation in Norway, 2011.Euro Surveill. 2012;17(50):20340.PMID: 23241234

11. European Centre for Disease Prevention and Control (ECDC). Measles case definition. 8 Sep 2012. Stockholm: ECDC. [Accessed 9 Jan 2015]. Available from: http://ecdc.europa. eu/en/activities/surveillance/euvac/case_definition/pages/ measles.aspx

12. El MubarakHS, De SwartRL, OsterhausADME, SchuttenM. Development of a semi-quantitative real-time RT-PCR for the detection of measles virus.J Clin Virol. 2005;32(4):313-7. DOI: 10.1016/j.jcv.2004.08.017 PMID: 15780811

13. WHO,. New genotype of measles virus and update on global distribution of measles genotypes.Wkly Epidemiol Rec. 2005;80(40):347-51.PMID: 16240986

14. RotaPA, BrownK, MankertzA, SantibanezS, ShulgaS, MullerCP, et al. Global distribution of measles genotypes and measles molecular epidemiology. J Infect Dis. 2011;204(Suppl 1):S51423. DOI: $10.1093 /$ infdis/jir118 PMID: 21666208

15. ThompsonJD, HigginsDG, GibsonTJ. CLUSTAL W: improving the sensitivity of progressive multiple sequence alignment through sequence weighting, position-specific gap penalties and weight matrix choice. Nucleic Acids Res. 1994;22(22):4673-80. DOI: $10.1093 / \mathrm{nar} / 22.22 .4673$ PMID: 7984417

16. Statens Serum Institut (SSI). Tema om Mæslinger. [Theme on measles]. Copenhagen: SSI. [Accessed 20 May 2015. Danish. Available from: http://www.ssi.dk/Aktuelt/Sygdomsudbrud/ Maeslingetema.aspx

17. MuscatM, SheferA, Ben MamouM, SpataruR, JankovicD, DeshevoyS, et al. The state of measles and rubella in the WHO European Region, 2013. Clin Microbiol Infect. 2014;20(Suppl 5):12-8. DOI: $10.1111 / 1469-0691.12584$ PMID: 24520948

18. World Health Organization (WHO) Regional Office for Europe. WHO Sub-Regional meeting for Measles/ Rubella National Reference Laboratories, Western and Central European Countries. 5-7 May 2014, Helsinki, Finland. Copenhagen: WHO Regional Office for Europe; 2014. Available from: http:// apps.who.int/iris/bitstream/10665/145671/1/WHO\%20 Subregional\%2omeeting $\% 2$ ofor $\% 2$ othe $\% 2$ measles $\% 20$ rubella\%2onational\%2oreference $\% 2$ 2olaboratories $\% 20$ of $\% 20$ western\%20and\%20central\%20European $\% 20$ Countries.pdf

19. GrothC, BottigerB, PlesnerA, ChristiansenA, GlismannS, HoghB. Nosocomial measles cluster in Denmark following an 
imported case, December 2008-January 2009. Euro Surveill. 2009;14(8):19126.PMID: 19250624

20. MuscatM, Hartvig ChristiansenA, BottigerBE, PlesnerA, GlismannS. A cluster of measles cases in Denmark following importation, January and February 2008. Euro Surveill. 2008;13(9):8050.PMID: 18445407

21. Muñoz-AlíaMA, Fernández-MuñozR, CasasnovasjM, PorrasMansillaR, Serrano-PardoÁ, Pagánl, et al. Measles virus genetic evolution throughout an imported epidemic outbreak in a highly vaccinated population. Virus Res. 2015;196:122-7. DOI: 10.1016/j.virusres.2014.11.015 PMID: 25445338

22. AllamMF. New measles vaccination schedules in the European countries?J Prev Med Hyg. 2014;55(1):33-4.PMID: 25916031

23. DefayF, De SerresG, SkowronskiDM, BoulianneN, OuakkiM, LandryM, et al. Measles in children vaccinated with 2 doses of MMR. Pediatrics. 2013;132(5):e1126-33. DOI: 10.1542/ peds.2012-3975 PMID: 24144708

24. WaaijenborgS, HahnéSJ, MollemaL, SmitsGP, BerbersGA, van der KlisFR, et al. Waning of maternal antibodies against measles, mumps, rubella, and varicella in communities with contrasting vaccination coverage. J Infect Dis. 2013;208(1):106. DOI: 10.1093/infdis/jit143 PMID: 23661802

25. De SerresG, Sciberras), NausM, BoulianneN, DuvalB,

RochetteL. Protection after two doses of measles vaccine is independent of interval between doses.J Infect Dis. 1999;180(1):187-90. DOI: 10.1086/314847 PMID: 10353877

26. VandermeulenC, MathieuR, GeertLR, PierreVD, KarelH. Longterm persistence of antibodies after one or two doses of MMR-vaccine.Vaccine. 2007;25(37-38):6672-6. DOI: 10.1016/j. vaccine.2007.07.008 PMID: 17692439

27. RosenJB, RotaJS, HickmanCJ, SowersSB, MercaderS, RotaPA, et al. Outbreak of measles among persons with prior evidence of immunity, New York City, 2011. Clin Infect Dis. 2014;58(9):120510. DOI: $10.1093 /$ cid/ciu105 PMID: 24585562

28. LeBaronCW, BeelerJ, SullivanBJ, ForghaniB, BiD, BeckC, et al Persistence of measles antibodies after 2 doses of measles vaccine in a postelimination environment. Arch Pediatr Adolesc Med. 2007;161(3):294-301. DOI: 10.1001/archpedi.161.3.294 PMID: 17339511

29. Ogbuanu IU, Kutty PK, Hudson JM, Blog D, Abedi GR, Goodell S, et al. Impact of a third dose of measles-mumps-rubella vaccine on a mumps outbreak. Pediatrics. 2012;130(6):e1567-74.

30. Krause TG, Valentiner-Branth $P$, Galle M, Mølbak K. Påmindelse om manglende børnevaccinationer, erfaringer efter de første fem måneder. [Reminder about missing childhood vaccinations, experiences from the first five months]. EPI-NYT week 42015. Cophenhagen: Statens Serum Institut; Danish. Available from: http://www.ssi.dk/Aktuelt/Nyhedsbreve/EPI-NYT/2015/ Uge \%204\%20-\%202015.aspx 